\title{
Adults with Communication Disabilities Experience Poorer Health and Healthcare Outcomes Compared to Persons Without Communication Disabilities
}

\author{
Michelle L. Stransky, PhD ${ }^{1,2}$, Kristin M. Jensen, MD, MSc ${ }^{3}$, and Megan A. Morris, PhD, MPH, CCC-SLP4 \\ 'Department of Community Health, Tufts University, Medford, MA, USA; ${ }^{2}$ Affiliated Research Faculty, Social Science Applied Research Center, \\ University of North Carolina, Wilmington, NC, USA; ${ }^{3}$ Departments of Pediatrics and Internal Medicine, University of Colorado School of Medicine, \\ Aurora, CO, USA; ${ }^{4}$ Adult and Child Consortium for Health Outcomes Research and Delivery Science (ACCORDS), Children's Hospital Colorado, \\ University of Colorado Anschutz Medical Campus, Aurora, CO, USA.
}

BACKGROUND: Persons with speech, language, and/or voice disabilities (collectively referred to as communication disabilities (CD)) represent 10\% of the US population, yet their healthcare outcomes have not been described. Generally, research shows that persons with disabilities have poorer health and healthcare outcomes than their non-disabled peers.

OBJECTIVES: To examine the health and healthcare outcomes of persons with CD compared to persons without CD. DESIGN: Retrospective cohort study of the 2012 National Health Interview Survey, which contained the first supplemental questions on CD. We compared proportional differences in outcomes; logistic and ordered logistic regression assessed the outcome measures across CD categories, controlling for demographics, noncommunication disabilities, and chronic conditions. Findings are weighted to permit national inferences.

PARTICIPANTS: Adults ( $\geq 18$ years old) were divided into 4 mutually exclusive groups: people with voice disabilities only; speech/language disabilities only; speech/language and voice disabilities; and people without CD.

MAIN MEASURES: Chronic health conditions; self-rated health; access to care; unmet needs for care; healthcare utilization.

KEY RESULTS: Adults with CD more frequently had $\geq 1$ chronic condition (voice $67.9 \%$, speech/language $68.6 \%$, speech/language and voice $79.9 \%$, no CD $50.1 \%$, $p<0.001$ ) and reported fair/poor health (voice $19.5 \%$, speech/language $32.5 \%$, speech/language and voice $48.3 \%$, no CD 11.2\%, $p<0.001$ ) compared to those without CD. Adults with CD more frequently utilized healthcare compared to those without CD. However, persons with $\mathrm{CD}$ endorsed greater difficulties accessing care than those without $\mathrm{CD}$, including identifying a usual source of care, trouble finding a physician, and delaying or foregoing care (e.g., delayed due to availability of care: voice $26.1 \%$, speech/language $37.2 \%$, speech/language and voice $30.8 \%$ no $\mathrm{CD} 16.1 \%, p<0.001)$.

Electronic supplementary material The online version of this article (https://doiorg/10.1007/s11606-018-4625-1) contains supplementary material. which is available to authorized users.

Received March 5, 2018

Revised July 2, 2018

Accepted August 1, 2018

Published online August 24, 2018
CONCLUSIONS: Persons with CD are medically complex and experience greater challenges accessing healthcare than persons without CD. Healthcare providers need support and tools to provide equitable care that addresses the medical needs of persons with $\mathrm{CD}$.

KEY WORDS: communication disabilities; chronic conditions; health care utilization; access to care; unmet needs for care.

J Gen Intern Med 33(12):2147-55

DOI: $10.1007 / \mathrm{s} 11606-018-4625-1$

๑) Society of General Internal Medicine 2018

\section{BACKGROUND}

Persons with speech, language, and/or voice disabilities (collectively referred to as communication disabilities (CD)) represent $10 \%$ of the US population. ${ }^{1} \mathrm{CD}$ can arise from a multitude of conditions (e.g., aphasia due to stroke, developmental stutter or dysfluency, aphonia from total laryngectomy) and result in patients having difficulty expressing and/or comprehending language. Given the rising rates of disability and the aging population, the proportion of persons with $\mathrm{CD}$ in the population is likely to increase. ${ }^{2}$

An expanding body of evidence suggests that people with disabilities experience poorer health and healthcare outcomes than those without disabilities, ${ }^{3,4}$ yet few studies to date have examined health and healthcare outcomes for adults with CD. In a study of Medicare recipients, Hoffman et al. reported low satisfaction with healthcare and patient-provider communication among persons with $\mathrm{CD}^{5}$ These findings have been echoed in qualitative studies describing poor patient-provider communication among patients with $\mathrm{CD}$ as a major contributor to low satisfaction. ${ }^{6-10}$ Bartlett et al. observed that patients with CD were three times more likely to experience adverse medical events during hospital stays compared to patients without CD. ${ }^{11}$ Outside of these reports, little is known about the overall health and healthcare outcomes of people with CD. Yet, healthcare providers need baseline information on these outcomes to address the needs of this growing population that reports poor experiences with healthcare delivery. 
In 2012, the National Health Interview Survey (NHIS) included supplemental questions about $\mathrm{CD} .^{12}$ This was the first health-related national survey to collect such information. Therefore, the purpose of this paper is to leverage this unique dataset to examine the health and healthcare outcomes of people with $\mathrm{CD}$ compared to people without $\mathrm{CD}$ in the USA. We pay particular attention to self-rated health, chronic conditions, and healthcare access and utilization. Based on the literature discussed above and our clinical experiences, we anticipate that people with $\mathrm{CD}$ will fare worse in these domains than their peers without $\mathrm{CD}$.

\section{METHODS}

\section{Data Source and Subjects}

For this retrospective cohort study, we used the 2012 National Health Interview Survey (NHIS). The NHIS is an annual inperson survey administered by the U.S. Census Bureau of community-dwelling civilians in households in the USA that addresses numerous health and healthcare topics. ${ }^{12}$ Surveyors asked a set of core survey questions on health conditions, behaviors, and healthcare access/utilization for each member of the sampled families. Additionally, they gathered information on one randomly sampled adult within each family unit. The NHIS is publicly available; the Institutional Review Board at Tufts University categorized this study as exempt from review.

The 2012 NHIS Sample Adult questionnaire contained the supplementary questions on CD. The Sample Adult file contains information on a total of 34,525 adults $\geq 18$ years old, representing a final response rate of $61.2 \% .^{12}$ Full information on the supplemental CD questions was available for 33,166 adults (96\%), which comprised the cohort used in this analysis.

\section{Communication Disabilities Definitions}

Respondents were categorized as having a communication disabilities (CD) based on an affirmative response to questions regarding difficulties or problems with their voice, speech, or language during the past 12 months. Respondents answering "no" to those all of these questions were categorized as not having a $\mathrm{CD}$. Respondents who refused or were uncertain were coded as missing $(n=49)$ and were excluded from the final analysis.

We created a mutually exclusive variable to define CD groups: people with (1) voice disabilities only (2) speech and/or language (SL) disabilities only, and (3) speech and/or language and voice (SLV) disabilities. We categorized voice disabilities separately from SL disabilities because the duration of these problems is significantly shorter (56 days in a year as compared to 142 and 195 days for respondents with speech or language disabilities). ${ }^{4}$ Consequently, it would be expected that voice disabilities are less likely to impact a respondent's health and health outcomes compared to speech or language disabilities.

\section{Study Outcomes}

Health. We examined respondents' self-reports of having 8 conditions: hypertension, stroke, emphysema, asthma, cancer, arthritis, diabetes, and cardiac disease. We summarized these reports into no conditions, 1 condition, or $\geq 2$ condition. Self-rated health was categorized as poor/fair, good, or very good/excellent.

Access to Healthcare. We included two measures of usual sources of care: having a usual place of care (1) when sick or in need of medical advice and (2) when routine or preventative care was needed. We coded respondents who reported the hospital Emergency Department as their usual source of care as not having usual sources of care.

Unmet Needs for Healthcare. Separate measures identify respondents who reported difficulty finding physicians or delaying or foregoing care due to cost. We categorized respondents as delaying care due to availability problems if they reported difficulty scheduling appointments, waiting too long to get an appointment or at the doctor's office, clinics not open when the respondent was available, or the respondent not having transportation to the clinic in the previous 12 months. Participants were coded as having foregone healthcare services if they reported being unable to afford prescription medications, mental healthcare, dental care, eyeglasses, specialist visits, or follow-up care.

Healthcare Utilization. Self-reported inpatient hospital stays, emergency department visits, and office-based visits over the past 12 months were measured using NHIS-defined categories. Inpatient hospital or emergency department visits were categorized into no stays/visits, 1 stay/visit, or more than 1 stay/visit because these were relatively rare events. The more frequently occurring outpatient visits were categorized into no visits, $1-3$ visits, or $\geq 4$ visits.

\section{Other Measures}

Self-reported race/ethnicity, marital status, educational attainment, sex, region of residence, and age were used as controls. Poverty status was measured as the family-sized adjusted income; publicly available imputations from the National Center for Health Statistics were used to account for non-response. Self-reported health insurance was categorized as uninsured, any private insurance, or public insurance only. We controlled for the following non-communication disabilities: physical, cognitive, visual, hearing, instrumental activities of daily living (IADL), and activities of daily living (ADL) disabilities.

\section{Data Analysis}

Analyses were conducted in 2016-2017 using Stata (version 11.0; Stata Corp.; College Station, TX) ${ }^{13}$ accounting for the 
Sample Adult weight and complex survey design. Statistical significance was defined as $p<0.05$. We examined the unadjusted association between outcomes and CD using Pearson's chi-squared test. Next, we used logistic and ordered logistic regressions (odds ratios (ORs)) to assess the outcome measures described above on $\mathrm{CD}$, controlling for demographics and noncommunication disabilities. Post-hoc analyses revealed that ordered logistic regressions violated the assumption of parallel lines; models that relaxed this assumption were substantively similar in magnitude, direction, and statistical significance. ${ }^{14}$ For ease of interpretation, we show the ordered logistic regression models here. For healthcare-related outcomes, we additionally controlled for chronic conditions. Other disability types were included as a control measure because a substantive body of literature has shown a significant overlap between these disability types and poor health and healthcare outcomes. ${ }^{15,16}$ Models including other disability types revealed few differences (details available upon request). We chose to present the models controlling for other disability types to more conservatively assess the impact of $\mathrm{CD}$ on outcomes.

\section{RESULTS}

Of the 33,166 adults included in the sample, $6.3 \%$ of adults endorsed voice disabilities, $2.2 \%$ endorsed SL disabilities, and $1.3 \%$ endorsed SLV disabilities (Table 1). People without CD and those with voice disabilities were more frequently mar-

Table 1 Characteristics of Persons with and Without Communication Disabilities

\begin{tabular}{|c|c|c|c|c|}
\hline & \multirow{2}{*}{$\begin{array}{l}\text { No comm. disability } \\
\%(n)\end{array}$} & \multirow{2}{*}{$\begin{array}{l}\text { Voice only disability } \\
\%(n)\end{array}$} & \multirow{2}{*}{$\begin{array}{l}\begin{array}{l}\text { Speech/language } \\
\text { (SL) disability }\end{array} \\
\%(n)\end{array}$} & \multirow{2}{*}{$\begin{array}{l}\begin{array}{l}\text { Speech/language + voice } \\
\text { (SLV) disability }\end{array} \\
\%(n)\end{array}$} \\
\hline & & & & \\
\hline Total $(n=33,166)$ & $90.2(29,820)$ & $6.3(2160)$ & $2.2(723)$ & $1.3(463)$ \\
\hline Female* & $51.1(16,429)$ & $66.2(1457)$ & $47.7(363)$ & $48.5(251)$ \\
\hline Age (years; mean $(\mathrm{SD}))^{*}$ & $46.4(17.7)$ & $48.8(18.8)$ & $45.0(19.2)$ & $49.6(20.9)$ \\
\hline $18-24$ & $12.7(2930)$ & $11.9(214)$ & $21.4(95)$ & $16.6(47)$ \\
\hline $25-34$ & $18.0(5451)$ & $15.7(324)$ & $14.2(113)$ & $11.2(48)$ \\
\hline $35-44$ & $17.3(5111)$ & $14.3(304)$ & $12.9(98)$ & $11.1(51)$ \\
\hline $45-54$ & $18.6(5146)$ & $18.8(351)$ & 16.8 (119) & $18.0(82)$ \\
\hline $55-64$ & $16.2(5016)$ & $16.4(386)$ & $17.5(146)$ & $18.4(102)$ \\
\hline$\geq 65$ & $17.2(6166)$ & $22.9(581)$ & $17.2(152)$ & $24.7(133)$ \\
\hline \multicolumn{5}{|l|}{ Race/ethnicity* } \\
\hline Hispanic & $15.3(5228)$ & $9.2(230)$ & $15.3(128)$ & $14.8(75)$ \\
\hline White, non-Hispanic & $66.8(17,872)$ & $76.6(1527)$ & $66.2(411)$ & $66.8(274)$ \\
\hline Black, non-Hispanic & $11.6(4457)$ & $10.8(312)$ & $14.0(148)$ & $14.4(91)$ \\
\hline Other, Non-Hispanic & $6.3(2263)$ & $3.4(91)$ & $4.5(36)$ & $4.0(23)$ \\
\hline Marital status* & $54.1(13,276)$ & $50.3(860)$ & 36.5 (214) & $37.1(133)$ \\
\hline Employment status* & $62.3(17,792)$ & $57.2(1148)$ & $38.3(274)$ & $35.9(143)$ \\
\hline \multicolumn{5}{|l|}{ Educational attainment* } \\
\hline Less than high school & $13.7(4642)$ & $11.6(279)$ & $22.0(194)$ & 27.9 (134) \\
\hline High school graduate & $26.2(7724)$ & $22.8(525)$ & $30.6(207)$ & $32.4(141)$ \\
\hline Some college & $20.4(5901)$ & $22.5(478)$ & $22.1(155)$ & $22.7(97)$ \\
\hline College degree & $39.8(11,553)$ & $43.1(878)$ & $25.3(167)$ & $17.1(91)$ \\
\hline \multicolumn{5}{|l|}{ Poverty status (imputed)* } \\
\hline$<100 \%$ FPL & $13.4(4993)$ & $12.8(373)$ & $24.6(213)$ & $25.4(135)$ \\
\hline 100-199\% FPL & $19.0(5412)$ & $19.3(406)$ & $25.8(167)$ & $27.9(112)$ \\
\hline 200-399\% FPL & $29.9(7552)$ & $30.5(574)$ & $27.6(166)$ & $30.3(110)$ \\
\hline$\geq 400 \%$ FPL & $37.7(8775)$ & $37.4(603)$ & $21.9(113)$ & $16.3(65)$ \\
\hline \multicolumn{5}{|l|}{ Health insurance* } \\
\hline Any private & $66.6(18,575)$ & $69.7(1397)$ & $49.0(312)$ & $44.5(192)$ \\
\hline Public only & $16.3(5830)$ & $17.7(465)$ & $32.2(271)$ & 39.9 (209) \\
\hline Uninsured & $17.0(5415)$ & $12.7(298)$ & $18.8(140)$ & $15.7(62)$ \\
\hline \multicolumn{5}{|l|}{ Region of residence* } \\
\hline Northeast & $18.4(5030)$ & 16.5 (339) & $16.6(100)$ & $11.9(54)$ \\
\hline Midwest & $22.3(6086)$ & $28.3(556)$ & $24.2(152)$ & $23.6(92)$ \\
\hline South & $36.5(10,870)$ & $36.1(757)$ & $37.3(279)$ & 39.7 (181) \\
\hline West & $22.8(7834)$ & $19.2(508)$ & $21.9(192)$ & $24.8(136)$ \\
\hline \multicolumn{5}{|c|}{ Non-communication disabilities } \\
\hline Any disability* & $14.8(5067)$ & $23.8(619)$ & $53.5(422)$ & $64.7(331)$ \\
\hline Physical* & $8.1(2956)$ & $15.8(416)$ & 29.7 (239) & $47.0(247)$ \\
\hline Cognitive* & 3.9 (1284) & $7.1(189)$ & $35.0(278)$ & 41.1 (199) \\
\hline Visual* & 2.9 (1018) & $5.7(146)$ & $10.1(86)$ & $23.6(113)$ \\
\hline Deaf or hard of hearing* & $4.5(1472)$ & $7.0(185)$ & $14.9(118)$ & $22.4(106)$ \\
\hline IADL* & $4.2(1516)$ & $7.7(219)$ & $25.8(201)$ & $37.5(193)$ \\
\hline ADL* & $2.1(735)$ & $4.4(114)$ & $14.0(96)$ & $27.4(128)$ \\
\hline
\end{tabular}

Poverty status was imputed by the National Center for Health Statistics. N's reflect the sample sizes that were not imputed

SL speech/language disability, SLV speech/language + voice disability, SD standard deviation, FPL federal poverty line, IADL instrumental activities of daily living, $A D L$ activities of daily living

$*_{p}<0.001$ 
ried, employed, and female than their counterparts with SL disabilities. Adults with SL and SLV disabilities more frequently reported public only health insurance and other cooccurring disabilities than did their peers without CD. Of note, $14.8 \%$ of persons without $\mathrm{CD}$ endorsed a non-communication disability vs. $23.8 \%, 53.5 \%$, and $64.7 \%$ of persons with Voice, SL, and SLV disabilities, respectively.

\section{Health Outcomes}

Adults with CD reported higher prevalence of any chronic conditions compared to persons without CD (Voice $67.9 \%$, SL $68.6 \%$, SLV 79.9\%, no CD 49.9\%, $p<0.001$; Table 2, eTable 1 ). Only $25 \%$ of adults without CD reported $\geq 2$ chronic conditions compared to $40 \%$ of people with Voice, $44 \%$ adults with SL, and $63 \%$ of adults with SLV disabilities $(p<0.001)$. Persons with CD were more likely to report additional chronic conditions compared to those without a $\mathrm{CD}$, controlling for the measures in Table 1 (AOR (95\% CI): Voice 1.9 (1.7-2.2), SL 1.7 (1.3-2.2), SLV 2.7 (1.9-3.8)).

Adults with CD were more likely to report fair/poor health compared to those without CD (Voice 19.5\%, SL 32.5\%, SLV 48.3\%; no CD 11.2\%; $p<0.001$; Table 2). After controlling for the characteristics in Table 1, adults with CD were less likely to rate their health highly compared to adults without CD (AOR (95\% CI): Voice 0.7 (0.6-0.8), SLV 0.5 (0.4-0.7)).

People across all CD types reported stroke, emphysema, and asthma more frequently than their peers without $\mathrm{CD}$ (eTable 1). Cardiovascular disease and diabetes were also more frequently reported by people with rather than without CD. Persons with Voice and SLV were 1.2-4.1 times more likely to report each of the specific chronic conditions com- pared to persons without $\mathrm{CD}$, controlling for the measures in Table 1 (Fig. 1). Similarly, persons with SL CD were 1.3-2.4 times more likely to report the same chronic conditions as those without CD.

\section{Healthcare Outcomes}

Adults with CD more frequently had $\geq 1$ hospital stays and $\geq 1$ emergency department visits than their peers without CD $(\geq 1$ hospital stay: Voice $14.0 \%$, SL 17.1\%, SLV 22.6\%, no CD $8.5 \% . p<0.001 ; \geq 1$ emergency department visits: Voice $27.5 \%$, SL $38.1 \%$, SLV 43.3\%, no CD $18.1 \%, p<0.001)$ (Table 3). Similarly, persons with CD more frequently had $\geq 1$ office-based visits compared to people without CD (Voice $91.0 \%$, SL $83.5 \%$, SLV 86.3\%, no CD 79.5\%, $p<0.001$ ) (Table 3 ). In analyses adjusted for the demographics and presence of non-communication disabilities described in Table 1, adults with $\mathrm{CD}$ had higher odds of more hospital stays (AOR (95\% CI): Voice $1.3(1.0-1.5))$ and emergency department visits (AORs (95\% CI): Voice 1.4 (1.2-1.6), SL 1.5 (1.1-1.9), SLV $1.5(1.1-2.0))$ compared to people without CD. Adults with Voice CD were $1.6(95 \%$ CI 1.4-1.8) times more likely to have additional office-based visits than their peers without $\mathrm{CD}$, accounting for the measures in Table 1. Persons with SL and SLV disabilities more frequently reported $\geq 4$ visits (SL 48.3\%; SLV $61.3 \%$ ) compared to people without CD $(34.8 \%, p<0.001)$. However, these findings were not significant in multivariate results.

All persons with $\mathrm{CD}$ in this cohort endorsed higher rates of "trouble finding a provider" for healthcare (Fig. 2; e Table 2), although the confidence intervals for the adjusted odds ratios were only significant for persons with Voice and SLV

Table 2 Disability, Number of Chronic Conditions, and Self-rated Health Among Persons with Communication Disabilities Compared to Persons Without Communication Disabilities $(n=33,166)$

\begin{tabular}{|c|c|c|c|c|c|c|c|c|c|c|}
\hline & \multirow{2}{*}{$\begin{array}{l}\text { No comm. } \\
\text { disabilities } \\
\% \\
\text { (n) }\end{array}$} & \multicolumn{3}{|c|}{ Voice only disability } & \multicolumn{3}{|c|}{$\begin{array}{l}\text { Speech/language (SL) } \\
\text { disability }\end{array}$} & \multicolumn{3}{|c|}{$\begin{array}{l}\text { Speech/language + voice (SLV) } \\
\text { disability }\end{array}$} \\
\hline & & $\begin{array}{l}\% \\
(n)\end{array}$ & $p$ value $^{\mathrm{a}}$ & $\begin{array}{l}\operatorname{AOR} \\
(95 \% C I)^{b}\end{array}$ & $\begin{array}{l}\% \\
(n)\end{array}$ & $p$ value $^{\text {a }}$ & $\begin{array}{l}\text { AOR } \\
(95 \% \text { CI })^{b}\end{array}$ & $\begin{array}{l}\% \\
(n)\end{array}$ & $p$ value $^{\text {a }}$ & $\operatorname{AOR}(95 \% \mathrm{CI})^{\mathrm{b}}$ \\
\hline \multicolumn{11}{|l|}{ Self-rated health ${ }^{\mathrm{c}}$} \\
\hline Fair/poor & $\begin{array}{l}11.2 \\
(3948)\end{array}$ & $\begin{array}{l}19.5 \\
(493)\end{array}$ & $<0.001$ & \multirow[t]{3}{*}{$\begin{array}{l}0.7 \\
(0.6-0.8)\end{array}$} & \multirow{2}{*}{$\begin{array}{l}32.5 \\
(263) \\
27.4 \\
(202)\end{array}$} & $<0.001$ & \multirow[t]{3}{*}{$\begin{array}{l}0.8 \\
(0.7-1.0)\end{array}$} & \multirow{2}{*}{$\begin{array}{l}48.3 \\
(241) \\
28.8 \\
(141)\end{array}$} & $<0.001$ & \multirow[t]{3}{*}{$\begin{array}{l}0.5 \\
(0.4-0.7)\end{array}$} \\
\hline Good & $\begin{array}{l}26.5 \\
(8275)\end{array}$ & $\begin{array}{l}28.0 \\
(639)\end{array}$ & 0.001 & & & $<0.001$ & & & $<0.001$ & \\
\hline Very good/excellent & $\begin{array}{l}62.3 \\
(17,597)\end{array}$ & $\begin{array}{l}52.5 \\
(1028)\end{array}$ & $<0.001$ & & $\begin{array}{l}40.1 \\
(258)\end{array}$ & $<0.001$ & & $\begin{array}{l}23.0 \\
(81)\end{array}$ & $<0.001$ & \\
\hline \multicolumn{11}{|c|}{ Number of chronic conditions ${ }^{\mathrm{c}}$} \\
\hline None & $\begin{array}{l}50.1 \\
(14,209)\end{array}$ & $\begin{array}{l}32.1 \\
(598)\end{array}$ & $<0.001$ & \multirow[t]{3}{*}{$\begin{array}{l}1.9 \\
(1.7-2.2)\end{array}$} & $\begin{array}{l}31.4 \\
(207)\end{array}$ & $<0.001$ & \multirow[t]{3}{*}{$\begin{array}{l}1.7 \\
(1.3-2.2)\end{array}$} & $\begin{array}{l}20.1 \\
(77)\end{array}$ & $<0.001$ & \multirow[t]{3}{*}{$\begin{array}{l}2.7 \\
(1.9-3.8)\end{array}$} \\
\hline One & $\begin{array}{l}25.0 \\
(7472)\end{array}$ & $\begin{array}{l}27.5 \\
(580)\end{array}$ & $<0.001$ & & $\begin{array}{l}24.5 \\
(177)\end{array}$ & 0.002 & & $\begin{array}{l}17.2 \\
(80)\end{array}$ & 0.014 & \\
\hline$\geq 2$ & $\begin{array}{l}24.9 \\
(8139)\end{array}$ & $\begin{array}{l}40.4 \\
(982)\end{array}$ & $<0.001$ & & $\begin{array}{l}44.1 \\
(339)\end{array}$ & $<0.001$ & & $\begin{array}{l}62.7 \\
(306)\end{array}$ & $<0.001$ & \\
\hline
\end{tabular}

SL speech/language disability, SLV speech/language + voice disability, AOR adjusted odds ratios, CI confidence interval

${ }^{a}$ Bivariate comparison of the communication disability group to the group with no communication disabilities

${ }^{b}$ Comparison of the communication disability group to the group with no communication disability, controlling for racelethnicity, marital status, employment status, educational attainment, poverty status, region of residence, sex, age, health insurance, and non-communication disabilities

${ }^{c}$ Number of chronic conditions and self-rated health were assessed using ordered logistic regression; adjusted odds ratios and 95\% CIs are shown 
Voice Disability

Speech/Language Disability

Speech/Language + Voice Disability

Any Chronic Condition

\section{Cardiovascular Disease}
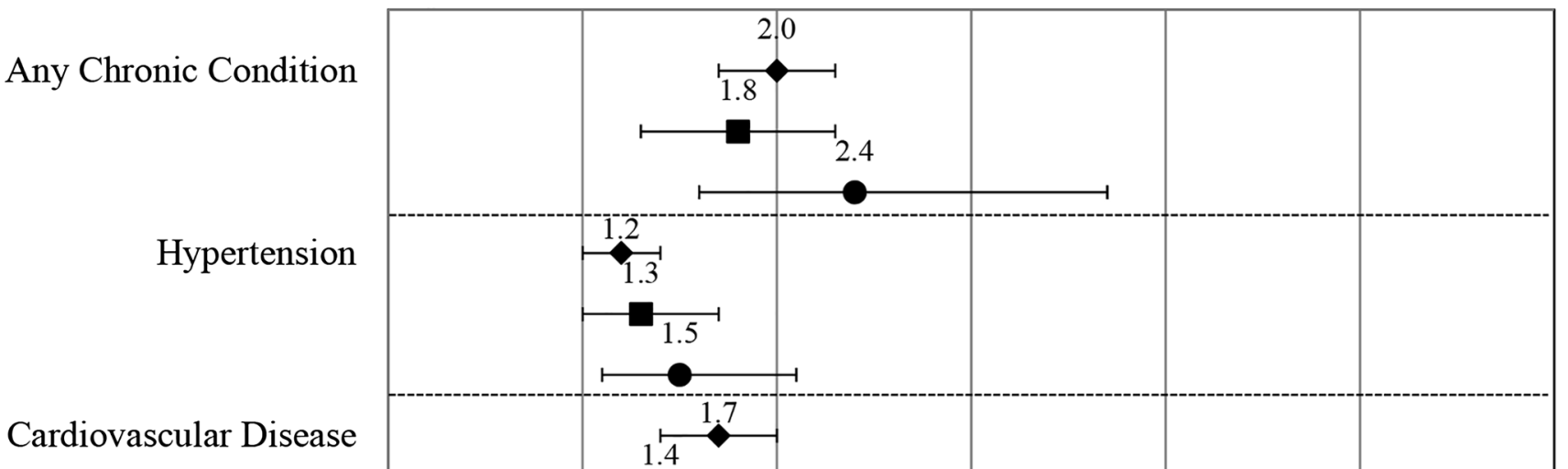

Hypertension

Stroke

Emphysema

Asthma
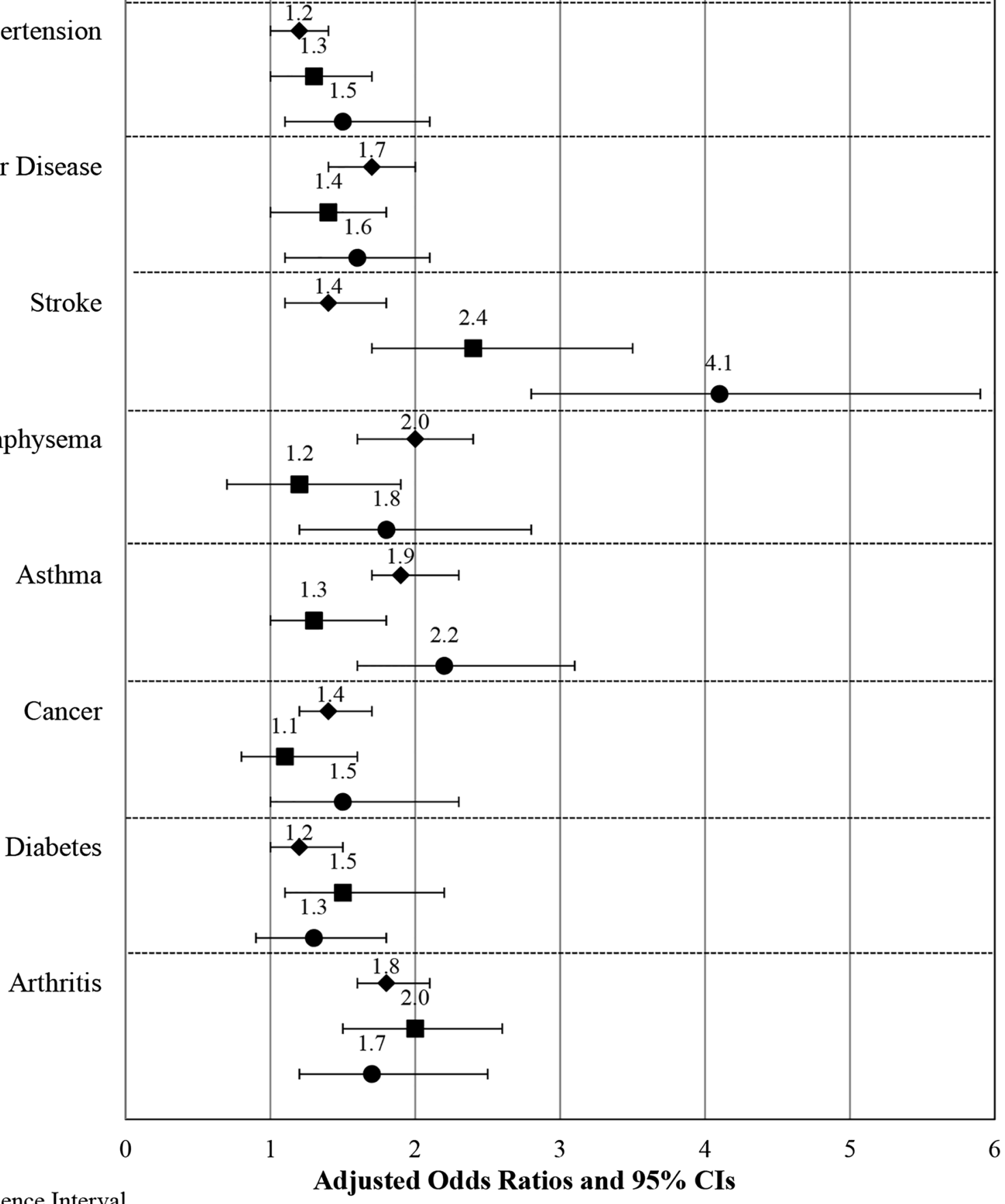

Note: $\mathrm{CI}=$ Confidence Interval

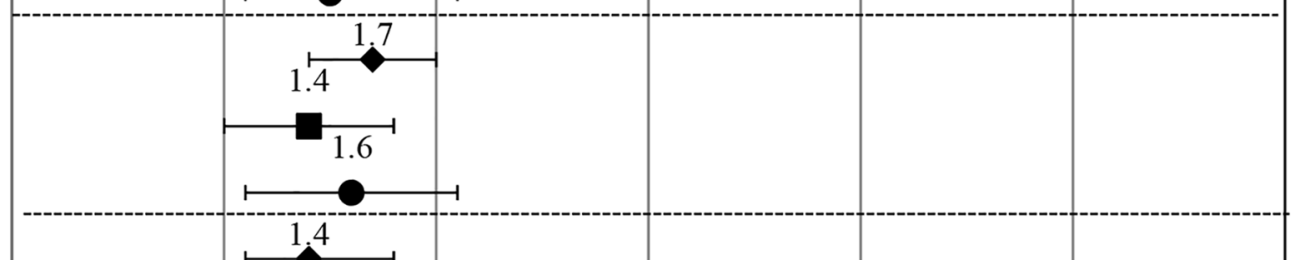

Odds ratios (and 95\% CIs) adjusted for for sex, age, race/ethnicity, marital status, employment status, educational attainment, poverty status, health insurance, region of residence, and non-communication disabilities.

Fig. 1 Forest plot of adjusted odds ratios (and 95\% CI) of chronic conditions by communication disability groups, compared to persons without communication disabilities 
Table 3 Healthcare Utilization for Persons with Communication Disorders Compared to Persons Without Communication Disorders $(n=$ 33,166)

\begin{tabular}{|c|c|c|c|c|c|c|c|c|c|c|}
\hline & \multirow{2}{*}{$\begin{array}{l}\text { No comm. } \\
\text { disorder } \\
\% \\
(n)\end{array}$} & \multicolumn{3}{|c|}{ Voice only disability } & \multicolumn{3}{|c|}{$\begin{array}{l}\text { Speech/language (SL) } \\
\text { disability }\end{array}$} & \multicolumn{3}{|c|}{$\begin{array}{l}\text { Speech/language + voice } \\
\text { (SLV) disability }\end{array}$} \\
\hline & & $\begin{array}{l}\% \\
(n)\end{array}$ & $p$ value $^{\mathrm{a}}$ & $\begin{array}{l}\text { AOR } \\
(95 \% \text { CI })^{b}\end{array}$ & $\begin{array}{l}\% \\
(n)\end{array}$ & $p$ value $^{\mathrm{a}}$ & $\begin{array}{l}\operatorname{AOR} \\
(95 \% \mathrm{CI})^{\mathbf{b}}\end{array}$ & $\begin{array}{l}\% \\
(n)\end{array}$ & $p$ value $^{\mathrm{a}}$ & $\begin{array}{l}\text { AOR } \\
(95 \% \text { CI })^{b}\end{array}$ \\
\hline \multicolumn{11}{|c|}{ Inpatient hospital stays } \\
\hline No stays & $\begin{array}{l}91.5 \\
(27,091)\end{array}$ & $\begin{array}{l}86.0 \\
(1834)\end{array}$ & $<0.001$ & $\begin{array}{l}1.3 \\
(1.0-1.5)\end{array}$ & $\begin{array}{l}82.9 \\
(594)\end{array}$ & $<0.001$ & $\begin{array}{l}1.1 \\
(0.8-1.6)\end{array}$ & $\begin{array}{l}77.4 \\
(342)\end{array}$ & $<0.001$ & $\begin{array}{l}1.2 \\
(0.8-1.7)\end{array}$ \\
\hline 1 stay & $\begin{array}{l}6.3 \\
(2001)\end{array}$ & $\begin{array}{l}9.4 \\
(211)\end{array}$ & $<0.001$ & & $\begin{array}{l}10.6 \\
(81)\end{array}$ & $<0.001$ & & $\begin{array}{l}11.3 \\
(58)\end{array}$ & $<0.001$ & \\
\hline$\geq 2$ stays & $\begin{array}{l}2.1 \\
(728)\end{array}$ & $\begin{array}{l}4.5 \\
(115)\end{array}$ & $<0.001$ & & $\begin{array}{l}6.5 \\
(48)\end{array}$ & $<0.001$ & & $\begin{array}{l}11.3 \\
(63)\end{array}$ & $<0.001$ & \\
\hline \multicolumn{11}{|c|}{ Emergency department visits } \\
\hline No visits & $\begin{array}{l}81.9 \\
(24,158)\end{array}$ & $\begin{array}{l}72.5 \\
(1505)\end{array}$ & $<0.001$ & $\begin{array}{l}1.4 \\
(1.2-1.6)\end{array}$ & $\begin{array}{l}61.9 \\
(442)\end{array}$ & $<0.001$ & $\begin{array}{l}1.5 \\
(1.1-1.9)\end{array}$ & $\begin{array}{l}56.6 \\
(256)\end{array}$ & $<0.001$ & $\begin{array}{l}1.5 \\
(1.1-2.0)\end{array}$ \\
\hline 1 visit & $\begin{array}{l}11.9 \\
(3648)\end{array}$ & $\begin{array}{l}15.9 \\
(381)\end{array}$ & $<0.001$ & & $\begin{array}{l}19.7 \\
(140)\end{array}$ & $<0.001$ & & $\begin{array}{l}17.2 \\
(82)\end{array}$ & $<0.001$ & \\
\hline$\geq 2$ visits & $\begin{array}{l}6.2 \\
(2014)\end{array}$ & $\begin{array}{l}11.6 \\
(274)\end{array}$ & $<0.001$ & & $\begin{array}{l}18.4 \\
(141)\end{array}$ & $<0.001$ & & $\begin{array}{l}26.2 \\
(125)\end{array}$ & $<0.001$ & \\
\hline \multicolumn{11}{|c|}{ Office-based visits } \\
\hline No visits & $\begin{array}{l}20.5 \\
(6193)\end{array}$ & $\begin{array}{l}9.0 \\
(200)\end{array}$ & $<0.001$ & $\begin{array}{l}1.6 \\
(1.4-1.8)\end{array}$ & $\begin{array}{l}16.5 \\
(122)\end{array}$ & $<0.001$ & $\begin{array}{l}1.0 \\
(0.8-1.2)\end{array}$ & $\begin{array}{l}13.7 \\
(49)\end{array}$ & $<0.001$ & $\begin{array}{l}1.1 \\
(0.8-1.5)\end{array}$ \\
\hline 1-3 visits & $\begin{array}{l}44.7 \\
(12,971)\end{array}$ & $\begin{array}{l}37.8 \\
(764)\end{array}$ & $<0.001$ & & $\begin{array}{l}35.2 \\
(231)\end{array}$ & 0.915 & & $\begin{array}{l}25.1 \\
(108)\end{array}$ & 0.452 & \\
\hline$\geq 4$ visits & $\begin{array}{l}34.8 \\
(10,656)\end{array}$ & $\begin{array}{l}53.3 \\
(1196)\end{array}$ & $<0.001$ & & $\begin{array}{l}48.3 \\
(370)\end{array}$ & $<0.001$ & & $\begin{array}{l}61.3 \\
(306)\end{array}$ & $<0.001$ & \\
\hline
\end{tabular}

SL speech/language disability, SLV speech/language + voice disability, AOR adjusted odds ratios; CI confidence interval

${ }^{a}$ Bivariate comparison of the communication disability group to the group with no communication disabilities

${ }^{b}$ Odds ratios comparing communication disability group to the group with no communication disability from ordered logistic regression analyses controlling for racelethnicity, marital status, employment status, educational attainment, poverty status, region of residence, sex, age, health insurance, chronic conditions, and non-communication disabilities

${ }^{c}$ Adjusted odds ratios from logistic regression comparing each of the communication disability groups to the group with no communication disability are shown in Fig. 2

disabilities endorsed similar experiences to persons without CD while persons with SLV disabilities had significantly greater difficulties identifying a usual source of care when ill (usual source of care when ill: AOR $(95 \% \mathrm{CI})$ : Voice $1.1(0.9$ 1.3)), SL 0.9 (0.6-1.2), SLV $0.5(0.3-0.9))$.

Persons with $\mathrm{CD}$ more frequently reported unmet needs for care than persons without $\mathrm{CD}$ (for example, delayed healthcare due to cost: Voice $16.4 \%$; SL: $17.0 \%$; SLV: $23.9 \%$; no CD: $10.4 \%, p<0.001$ for pairwise comparisons; delayed due to availability: Voice: $18.9 \%$; SL: $25.2 \%$; SLV: $30.8 \%$; no CD: $9.4 \%, p<0.001$ for pairwise comparisons). All CD groups reported greater unmet needs for care, with adjusted odds ranging from 1.6 (95\% CI 1.3-1.9, foregone medical care due to cost) to 2.3 (95\% CI 1.7-3.1, delayed care due to availability).

\section{DISCUSSION}

This study provides the first descriptions of health and healthcare outcomes among persons with CD in the U.S. We observed that persons with $\mathrm{CD}$ are generally more medically complex and have greater challenges accessing care than people without $\mathrm{CD}$, despite controlling for demographics, other non-communication disability types, and chronic conditions.
Persons with $\mathrm{CD}$ were as likely as those without $\mathrm{CD}$ to have usual sources of care for routine care and illness. Despite this, they frequently reported unmet healthcare needs due to cost, lack of availability, and difficulty finding healthcare providers. These challenges may be related to the fact that this is an unhealthy population with low financial resources and high rates of public insurance, which is not accepted by all providers. Communication barriers amplify these difficulties. Patients with hearing disabilities report challenges scheduling appointments over the phone. ${ }^{17}$ Similarly, patients with CD report frequently switching healthcare providers as a consequence of communication challenges with providers and staff. ${ }^{6}$ The negative effects of communication challenges are unsurprising given that it is well-documented that people with limited English proficiency, a population that also presents with communication challenges, also face multiple barriers to accessing care. ${ }^{18}$

Persons with CD have high rates of healthcare utilization, despite reporting difficulty finding providers and having unmet care needs. Approximately $50 \%$ of people with CD reported having $\geq 4$ office-based visits in the previous year. A quarter of those with SLV disabilities had $\geq 2$ emergency department visits and $22 \%$ had $\geq 1$ inpatient hospital stay in the past year. The frequency of interactions with healthcare settings highlights the need for healthcare providers and systems to be prepared for communicating with and providing care for patients with $\mathrm{CD}$. 


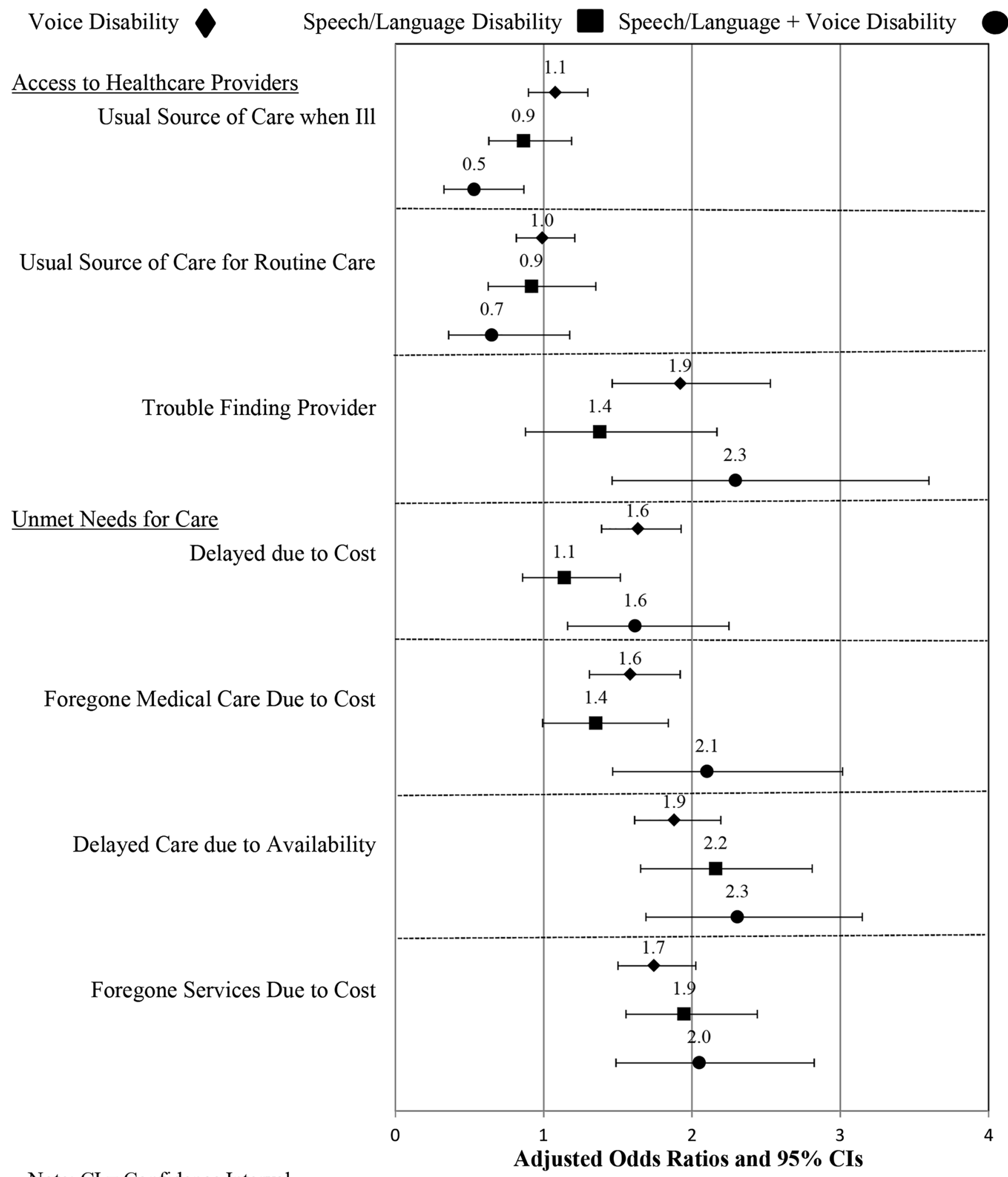

Note: $\mathrm{CI}=$ Confidence Interval

Odds ratios (and 95\% CIs) adjusted for for sex, age, race/ethnicity, marital status, employment status, educational attainment, poverty status, region of residence, chronic conditions and non-communication disabilities.

Fig. 2 Forest plot of adjusted odds ratios (and 95\% CI) of access to care by communication disability groups, compared to persons without communication disabilities

Despite previous studies that show that voice disabilities are less severe than SL disabilities, we found that adults with voice disabilities, both alone and accompanied by SL disabilities, were more likely to report chronic conditions and greater 
challenges accessing care compared to those without $\mathrm{CD}$. This is a critical finding, as $7 \%$ of the US population reports voice disabilities. ${ }^{1}$ Other studies have found that persons with voice disabilities report lower quality of life and health compared to those without voice disabilities. ${ }^{19}$ Further research is needed to understand what factors contribute to lower quality of health care, health, and access for this relatively large portion of the US population.

The results of this study are consistent with research findings in other disability populations. A study comparing people without disabilities to people with either cognitive or physical disabilities found that those with disabilities had higher rates of chronic conditions and were less likely to rate their health as good; however, they were more likely to have usual sources of care. ${ }^{15}$ While the prevalence of co-occurring disabilities among the group of people with $\mathrm{CD}$ is high, we found that disparities in health and healthcare outcomes based on CD persisted compared to people without CD. This suggests that there is something particular about $\mathrm{CD}$ that contributes to differences in health and healthcare utilization.

Providing care for patients with $\mathrm{CD}$ is inherently difficult due to communication challenges. The Joint Commission names those with $\mathrm{CD}$ as a population at high risk for ineffective communication and calls for healthcare systems and clinicians to provide additional supports to ensure effective communication and high-quality healthcare. ${ }^{20}$ Additionally, Section 1557 of the Patient Protection and Affordable Care Act mandates that hospitals and clinics provide patients with disabilities "auxiliary aids and services" to ensure equitable access to healthcare communication. ${ }^{21}$ Unfortunately, providers receive little, if any, training or support in this area. ${ }^{22}$ Clinical encounters with people with CD often take longer (due to communication barriers and the fact that they have higher rates of multiple chronic conditions) and tools and strategies to ensure effective communication may not be evident. For example, research has shown that people with $\mathrm{CD}$ have improved comprehension and participation in conversations when communication partners use strategies such as writing down key words while speaking or using visual aids. ${ }^{23}$ Yet, a study of video-recorded clinical encounters revealed that clinicians rarely, if ever, used evidence-based communication strategies with patients with language disabilities. ${ }^{8}$ Providing clinicians the right supports and tools to effectively communicate with patients with $\mathrm{CD}$ has the potential to significantly improve the quality of care provided to this vulnerable population.

The 2012 NHIS was the first national survey of health and healthcare to specifically query $\mathrm{CD}$. Our results reveal the presence of significant disparities in health and healthcare delivery among persons with $\mathrm{CD}$ and strongly suggests that questions about $\mathrm{CD}$ be included on future national surveys. This will support longitudinal tracking, which is critical to identifying trends in health and healthcare outcomes for patients with $\mathrm{CD}$ and supporting positive health and healthcare outcomes.

\section{Limitations}

The NHIS is cross-sectional by design and, thus, it is not possible to determine the causality of chronic conditions and the presence of CD. Certain CD are more likely to result from chronic conditions. For example, the high rates of stroke in those with SL disabilities is not surprising given that approximately 1 million people in the USA are living with aphasia, a language disorder most often caused by stroke. ${ }^{24}$ Regardless of the cause of the specific $\mathrm{CD}$, we found that the majority of those with CD (68 to $80 \%$ ) live with at least one chronic condition that requires continued medical intervention. Therefore, providing effective healthcare that is sensitive to the needs of this population is paramount to ensuring that patients with $\mathrm{CD}$ achieve the most optimal health possible.

\section{CONCLUSIONS}

Persons with CD are an understudied and overlooked population in our healthcare system, even though they represent $10 \%$ of the US population, have high rates of healthcare utilization, and experience significant disparities in health and healthcare outcomes. Moving the needle to improve the health of our nation necessitates that our healthcare providers and systems be prepared to provide high-quality patient-centered healthcare to patients with CD.

Corresponding Author: Michelle L. Stransky, PhD; Department of Community Health Tufts University, 574 Boston Ave., Suite 208, Medford, MA 02155, USA (e-mail: Michelle.stransky@tufts.edu).

\section{Compliance with Ethical Standards:}

Conflict of Interest: The authors declare that they do not have a conflict of interest.

\section{REFERENCES}

1. Morris MA, Meier SK, Griffin JM, Branda ME, Phelan SM. Prevalence and etiologies of adult communication disabilities in the united states: Results from the 2012 national health interview survey. Disability and Health Journal. 2016;9(1):140-144.

2. Institute of Medicine. Workshop on disability in america: A new look: Summary and background papers. Washington, DC: The National Academies Press; 2006.

3. Havercamp SM, Scandlin D, Roth M. Health disparities among adults with developmental disabilities, adults with other disabilities, and adults not reporting disability in north carolina. Public Health Rep. 2004;119(4):418-426.

4. Krahn GL, Walker DK, Correa-De-Araujo R. Persons with disabilities as an unrecognized health disparity population. Am J Public Health. 2015;105(S2):S198-S206.

5. Hoffman JM, Yorkston KM, Shumway-Cook A, Ciol MA, Dudgeon BJ, Chan L. Effect of communication disability on satisfaction with health care: A survey of medicare beneficiaries. American Journal of SpeechLanguage Pathology. 2005; 14(3):221-228.

6. Morris MA, Dudgeon BJ, Yorkston K. A qualitative study of adult AAC users' experiences communicating with medical providers. Disability and Rehabilitation: Assistive Technology. 2013;8(6):472-481.

7. Murphy J. Perceptions of communication between people with communication disability and general practice staff. Health Expectations. 2006;9(1):49-59. 
8. Morris MA, Clayman ML, Peters KJ, Leppin AL, LeBlanc A. Patientcentered communication strategies for patients with aphasia: Discrepancies between what patients want and what physicians do. Disability and health journal. 2015;8(2):208-215.

9. Nicolaidis C, Raymaker D, McDonald $\mathbf{K}$, et al. Comparison of health care experiences in autistic and non-autistic adults: A cross-sectional online survey facilitated by an academic-community partnership. Journal of general internal medicine. 2013;28(6):761-769.

10. Nordehn G, Meredith A, Bye L. A preliminary investigation of barriers to achieving patient-centered communication with patients who have stroke-related communication disorders. Topics in Stroke Rehabilitation. 2006;13(1):68-77.

11. Bartlett G, Blais R, Tamblyn R, Clermont RJ, MacGibbon B. Impact of patient communication problems on the risk of preventable adverse events in acute care settings. CMAJ. 2008;178(12):1555-1562.

12. National Center for Health Statistics. Data file documentation, national health interview survey, 2012 (machine reaable data file and documentation). Hyattsville: National Center for Health Statistics, Centers for Disease Control and Prevention; 2013.

13. StataCorp L. Stata 15.1 for windows. . 2017.

14. Williams R. Generalized order logit/partial proprotional odds models for ordinal dependent variables. The Stata Journal. 2006;6(1):58-82.

15. Reichard A, Stolzle H, Fox MH. Health disparities among adults with physical disabilities or cognitive limitations compared to individuals with no disabilities in the united states. Disability and Health Journal. 2011;4(2):59-67.

16. Reichard A, Gulley SP, Rasch EK, Chan L. Diagnosis isn't enough: Understanding the connections between high health care utilization, chronic conditions and disabilities among US working age adults. Disability and health journal. 2015;8(4):535-546.

17. Iezzoni LI, O'Day BL, Killeen M, Harker H. Communicating about health care: Observations from persons who are deaf or hard of hearing. Ann Intern Med. 2004;140(5):356-362.

18. Fiscella K, Franks P, Doescher MP, Saver BG. Disparities in health care by race, ethnicity, and language among the insured: Findings from a national sample. Med Care. 2002;40(1):52-59.

19. Merrill RM, Anderson AE, Sloan A. Quality of life indicators according to voice disorders and voice-related conditions. Laryngoscope. 2011;121(9):2004-2010.

20. The Joint Commission. Advancing effective communication, cultura competence, and patient- and family-centered care: A roadmap for hospitals. Oakbrook Terrace: The Joint Commission; 2010.

21. Nondiscrimination in health programs and activities, final rule. Fed Regist. 2016;81(96):31375-31473.

22. Burns MI, Baylor CR, Morris MA, McNalley TE, Yorkston KM. Training healthcare providers in patient-provider communication: What speechlanguage pathology and medical education can learn from one another. Aphasiology. 2012;26(5):673-688.

23. Simmons-Mackie N, Raymer A, Armstrong E, Holland A, Cherney LR Communication partner training in aphasia: A systematic review. Arch Phys Med Rehabil. 2010;91(12):1814-1837.

24. National Institute on Deafness and Other Communication Disorders. Aphasia. https://www.nidcd.nih.gov/health/aphasia. Updated 2017. 